\title{
SYNCHRONIC BAND AND ITS IMPLICATION IN THE COMETARY DUST
}

\author{
Jun-1ch1 Watanabe [1] \& Kimihiko N1shioka [2] \\ 1: National Astronomical observatory of Japan, \\ Mitaka, Tokyo, 181, Japan \\ 2: Olympus Optical Company Ltd., \\ 1-43-2, Hatagaya, Shibuya-ku, Tokyo, 181, Japan
}

\begin{abstract}
The unlque morphology of the synchronic band in the cometary dust tail is explained by finite-lifetime fragment model (Nishioka and Watanabe, 1990). However, this model needs a severe restriction on the lifetime of the dust fragments;25-70 days $(r=1$ A.U. $)$. This implies that detailed analysis of the synchronic band may reveal physical properties of the cometary dust particles. In this paper, we suggest that the fragments in the synchronic band are relatively pure ice if they are not organic grains.
\end{abstract}

\section{Introduction}

The narrow striae have been observed in the dust tail of several large comets. These striae are called "synchronic bands"(SYBs hereafter). The formation of SYBs is an interesting subject in cometary dust researches. Nishioka and Watanabe(1990) studied the SYBs formation on the basis of finite lifetime fragment model(FLM hereafter), which solved two problems remained in the proposed models so far. One is why the parent particles simultaneously break up at a certain time. Although Sekanina and Farrell(1980) succeeded to simulate the morphology of SYBs with an assumption of the simultaneous fragmentation, the physical reason of this assumption is not clear. Another problem is that the observed length of SYBs was not zero even if the observed epoch was the time before the estimated fragmentation. These two problems were solved by the FLM described in the next section.

Although the FLM model succeeded to simulate the morphology of SYBs, It needs a severe restriction on the lifetime of the fragmented dust particles. This paper introduces some analyses of SYBs in several comets on the basis of the FLM, and deals with the physical properties of the cometary dust particles in the SYBs.

A.C. Levasseur-Regourd and H. Hasegawa (eds.), Origin and Evolution of Interplanetary Dust, 253-256. (C) 1991 Kluwer Academic Publishers, Printed in Japan. 


\section{Finite Lifetime Fragment Model}

The FLM for SYBs is described schematically in figure 1 . Instead of the one-time, simultaneous fragmentation, parent particles break up continuously. All the created fragments are assumed to have a finite lifetime $\tau_{s}$. This lifetime is reduced to the value at the heliocentric distance of $1 \mathrm{~A} . \mathrm{U}$. This assumption means that the fragments cannot be observed when the elapsed time from the fragmentation is longer than the lifetime $\tau_{s}$. The elapsed time in the solar radiation of the fragments $\tau$ f is calculated by

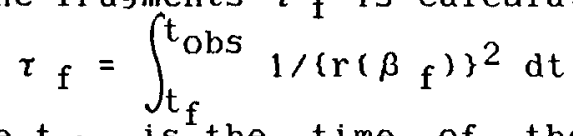

where $t_{\text {obs }}$ is the time of the observation, $t_{f}$ is the fragmentation time, $r\left(\beta_{f}\right)$ is the heliocentric distance of the fragment, $\beta f$ the ratio of the radiation pressure to the gravitation. The fragments, of which the elapsed time $\tau$ f is less than $\tau$, survive as the scattering materials in the optical wavelength.

The merit of the FLM is that we do not need unnatural assumption of the simultaneous fragmentation of the parent particles, or of other complicated mechanisms for the dust particles acceleration proposed so far. This model explains not only the observed width of SYBs, but also the length of SYBs by using an appropriate lifetime of the fragments. The lifetime is determined to reproduce the observed morphology of SYBs using equation (1). On the basis of this model, Nishioka and Watanabe(1990) derived the lifetime of the fragments in the SYBs of three comets $133.6-43.0$ days for Comet Seki-Lines 1962III; 23.0-67.1 days for Comet Mrkos 1957IX; 28.0-54.0 days for Comet west 1976VIl. The lifetime of the fragments lies between 25-70 days for all SYBs. This finite lifetime of 25-70 days gives us a hint for discussing the physical properties of the dust particles.

\section{Implication for the Cometary Dust in Synchronic Bands}

The parent particles of SYBs must be large in size, and be fragile. It should be noted that these SYBs appeared only in the new comets in the oort sense. Hence, the parent particles may contain more volatile materials. Then, there may be a lot of water ice grains in the fragments of SYBs. Mukai (1986) calculated the lifetime of both pure and dirty water ice grains at 1 A.U. as shown in figure 2. The 1 if etime of the heterogeneous grains is less than $1000 \mathrm{sec}$ even if the size is about $1 \mathrm{~mm}$. On the other hand, the lifetime of the pure ice grains is more than 1000 days. The calculated dirty grains consisted of amorphous water ice with magnetite and silicate inclusions. The volume fraction of the inclusions is 0.1 . Even if the volume fraction of 


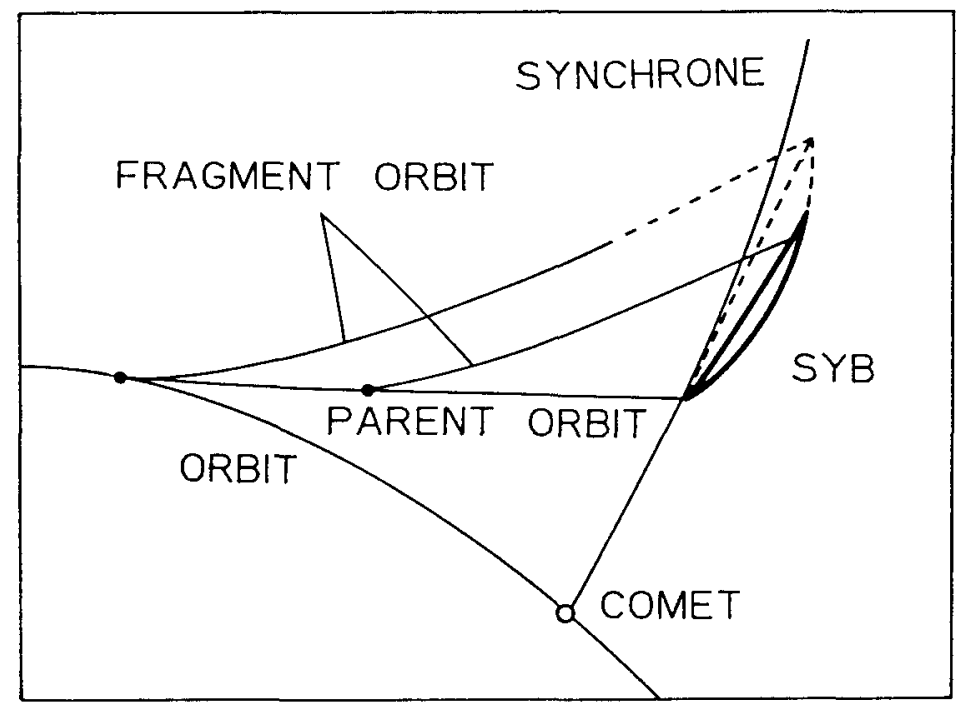

Figure 1. Formation of SYB on the basis of the finite lifetime fragment model(FLM). Parent particles ejected from the nucleus break up continuously. Fragment created earlier time vanish before the time of observation, and cannot be seen as indicated by the dashed line.

Figure 2. Lifetime of released icy grains with radius $s$ at heliocentric distance of 1 A.U. derived by Mukai(1986). The lifetime of fragments in SYBs derived by this study is shown by two thin lines, which may suggest that they are relatively pure icy grains.

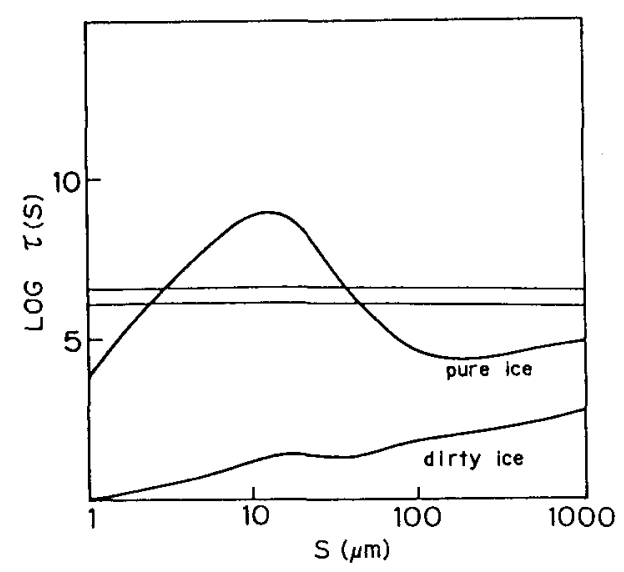


the magnetite was 0.001 , Mukai(1986) indicated that the iffetime of the dirty ice would not change. However, if fraction of the inclusion of the absorbing material in the water-ice grains is less than 0.001 , or if the inclusion is some kinds of organic materials, then the lifetime may be close to that of the pure 1ce grains. Therefore, the observed lifetime of 25-70 days may be due to the relatively pure lce grains ejected by the violent jets from the deep location in the cometary nucleus. Because the epoch of the parent particle ejection for SYBs was near the perihelion passage, these particles may have been pure materials ejected from the deep location.

Another possible candidate for the fragments in SYBs is organic grain. Although the CHON particles detected by the spacecrafts of Halley's mission may be a candidate, the lack of information on the optical properties prevented us from discussing further detalis. The optical constants of organic grain "tholin" are studied by Khare et al.(1984). Lamy and Perrin(1988) derived the optical properties, and concluded that the organic grains have a potential for explaining several puzzling cometary observations such as gaseous jets. Although the lifetime of the tholin itself is shorter by an order than 25-70 days, we cannot deny other organic materials as candidates for the fragments in SYBs.

\section{References}

Akabane, T. (1983) 'The secondary tail of Comet 1976VI West', Pub. Astron. Soc. Japan, 35, 565-578.

Khare, B.N., Sagan, C., Arakawa, E.T., Suits. F., Callcott, T.A., and Williams, M.W. (1984) 'Optical constants of organic Tholins produced in a simulated Titanian atmosphere:From soft $\mathrm{X}$-ray to mlcrowave frequencies'. Icarus, 60, 127-137.

Lamy, P.L., and Perrin, J.-M. (1988) 'Optical properties of organic grains:Implications for interplanetary and cometary dust', Icarus, 76, 100-109.

Mukai, T. (1986) 'Analysis of a dirty water-1ce model for cometary dust', Astron. Astrophys., 164, 397-407.

Nishioka, K., and Watanabe, J. (1990) 'Finite lifetime fragment model for synchronic band formation in dust tails of comets', Icarus, 87, 403-411.

Sekanina, Z., and Farrell, J.A. (1980) 'The striated dust tal1 of Comet West 1976VI as a particle fragmentation phenomenon ' Astron. J., 85, 1538-1554. 